\title{
Growth and properties of lead iodide thin films by spin coating
}

\author{
D ACUÑ $A^{1}$, B KRISHNAN ${ }^{1,2, *}$, S SHAJI ${ }^{1,2}$, S SEPÚLVEDA $^{1,2}$ and J L MENCHACA ${ }^{3}$ \\ ${ }^{1}$ Facultad de Ingeniería Mecánica y Eléctrica, Universidad Autónoma de Nuevo León, San Nicolás de los Garza, Nuevo \\ León 66455, México \\ ${ }^{2}$ Universidad Autónoma de Nuevo León, Centro de Innovación, Investigación y Desarrollo de Ingeniería y Tecnología, \\ Apodaca, Nuevo León 66600, México \\ ${ }^{3}$ Facultad de Ciencias Físico Matemáticas, Universidad Autónoma de Nuevo León, San Nicolás de los Garza, Nuevo León \\ 66455, México
}

MS received 19 July 2015; accepted 21 March 2016

\begin{abstract}
In this study, lead iodide $\left(\mathrm{PbI}_{2}\right)$ thin films were deposited on glass substrates by spin coating a solution of $0.2 \mathrm{M} \mathrm{PbI}_{2}$ dissolved in dimethylformamide, varying the deposition time and the spin speed. The thickness of the thin films decreased with increase in spin speed and deposition time, as examined by profilometry measurements. The structure, morphology, optical and electrical properties of the thin films were analysed using various techniques. $\mathrm{X}$-ray diffraction patterns revealed that the thin films possessed hexagonal structures. The thin films were grown highly oriented to [001] direction of the hexagonal lattice. Raman peaks detected at 96 and $136 \mathrm{~cm}^{-1}$ were corresponding to the characteristic vibration modes of $\mathrm{PbI}_{2}$. The $\mathrm{X}$-ray photoelectron spectroscopy detected the presence of $\mathrm{Pb}$ and $\mathrm{I}$ with core level binding energies corresponding to that in $\mathrm{PbI}_{2}$. Atomic force microcopy showed smooth and compact morphology of the thin films. From UV-Vis transmittance and reflectance spectral analysis, the bandgap of the thin films $\sim 2.3 \mathrm{eV}$ was evaluated. The dark conductivity of the thin films was computed and the value decreased as the deposition time and spin speed increased.
\end{abstract}

Keywords. Lead iodide thin films; spin coating; structure and morphology; XRD; Raman spectrum.

\section{Introduction}

Lead iodide $\left(\mathrm{PbI}_{2}\right)$ belongs to the layered semiconductor family, and is a widely studied semiconductor for various applications such as X-ray detectors [1], medical imaging [2], nuclear detection [3] and photosensitivity semiconductormetal applications [4]. It has also been used as a precursor for obtaining the methylammonium lead iodide perovskite-based solar cell [5], one of the highest solution-processed solar cells reported so far. Lead iodide exists in hexagonal crystalline structure having an intrinsic bandgap of 2.3-2.6 eV, depending on the deposition method [6]. The basic structure of $\mathrm{PbI}_{2}$ is a layer of $\mathrm{Pb}$ atoms sandwiched between two layers of I atoms. The basic building block of the crystal structure is a near-octahedral $\left[\mathrm{PbI}_{6}\right]^{4-}$ unit, in which each $\mathrm{Pb}$ atom is surrounded by $6 \mathrm{I}$ atoms. Interactions between the layers are of weak Van der Waals type; so, there are many ways of stacking the layers and hence many polytypes exist for this compound [7].

Thin films of lead iodide can be obtained by a variety of simple and low-cost solution methods. One of them was the iodination of chemical bath-deposited $\mathrm{PbS}$ thin films

\footnotetext{
*Author for correspondence (kbindu_k@yahoo.com,
} krishnan.bindu@uanl.edu.mx) in a dark chamber containing $\mathrm{I}_{2}$ crystals. Even though this method was simple and required low temperatures, the impurities of $\mathrm{PbS}$ were found in the $\mathrm{PbI}_{2}$ thin films under certain conditions [8]. Another solution process to deposit $\mathrm{PbI}_{2}$ was by dissolving lead iodide powder in water at $100^{\circ} \mathrm{C}$ up to the solubility limit, followed by slow cooling to deposit lead iodide platelets on the substrates. The layers were of hexagonal crystal structured oriented along [001] direction, and were photoconductive [9]. Other techniques such as sol-gel method [10] and spray pyrolysis [11] were also used. Among the methods to obtain thin films, spin coating offers a lowcost and facile method to obtain good quality thin films. Spin coating is simple because the spin parameters such as the deposition time and spin speed can easily be controlled to tune the final film thickness. Also, concentration of the precursor, temperature and properties of the solvent are crucial parameters to control the quality of the thin films [12]. However, a systematic study on the properties of $\mathrm{PbI}_{2}$ thin films deposited by this method is yet to be done.

In this study, we use spin coating to prepare $\mathrm{PbI}_{2}$ thin films on bare glass substrates and ITO-coated glass (glass/ITO) substrates. Spin speed and deposition time were varied to obtain the thin films of different thickness. A systematic study on the effect of variation in the deposition parameters on the structural, optical and electrical properties of the films is presented. 


\section{Experimental}

\subsection{Thin film preparation}

$\mathrm{PbI}_{2}$ powder was prepared by the reaction between solutions containing $1 \mathrm{M} \mathrm{Pb}\left(\mathrm{NO}_{3}\right)_{2}$ (Fermont, $99.6 \%$ purity) and $2 \mathrm{M}$ KI (Fermont, 99.3\% purity). The yellow powder was rinsed with deionized water and left overnight to dry, and used without any further purification.

Thin films of $\mathrm{PbI}_{2}$ were deposited as follows: drops of $0.2 \mathrm{M}$ solution of $\mathrm{PbI}_{2}$ dissolved in dimethylformamide (J.T. Baker, $99.96 \%$ purity) was dispensed on well-cleaned glass substrates. The drops were spinned to form $\mathrm{PbI}_{2}$ thin film using a Spin Coater (Laurel Technologies, Model WA400BZ-\&NPP/Lite). The thin films were prepared by varying the spin speed in the range of 3000-7000 r.p.m. and the deposition time from 15 to $35 \mathrm{~s}$. Two set of samples were prepared: (a) by varying the spin speed $3000,4000,5000$, 6000 and 7000 r.p.m., labelled as SS3000, SS4000, SS5000, SS6000 and SS7000, respectively, deposited for $25 \mathrm{~s}$ and (b) by varying duration of deposition for 15, 20, 25, 30 and $35 \mathrm{~s}$ labelled as DT15, DT20, DT25, DT30 and DT35, respectively, keeping the spin speed at 7000 r.p.m. All the samples were heated in a vacuum oven (T M Vacuum Products, $10^{-3}$ torr) at $100^{\circ} \mathrm{C}$ for $1 \mathrm{~h}$ to evaporate the solvent.

\subsection{Characterization}

The crystalline structure of these thin films was analysed using an X-ray diffractometer (Rigaku D-Max) employing $\mathrm{CuK} \alpha_{1}$ radiation $(\lambda=1.54056 \AA)$. Phase identification was confirmed by the Raman spectra of the thin films using a Raman microscope (Thermo Scientific DXR) with $532 \mathrm{~nm}$ excitation wavelength. Elemental composition and the chemical states were detected using an X-ray photoelectron spectrometer (Thermo Scientific K-Alpha) with monochromatized AlK $\alpha$ radiation $(h v=1486.68 \mathrm{eV})$. The morphology of the thin films was studied using an atomic force microscope (Solver Pro NT-MDT). The thickness of the films was determined by profilometry (KLA Tencor Alpha Step D-100 profilometer).

Table 1. Preparation conditions of $\mathrm{PbI}_{2}$ thin films and their thicknesses.

\begin{tabular}{|c|c|c|}
\hline \multicolumn{2}{|c|}{ Sample conditions } & \multirow[b]{2}{*}{ Thickness (nm) } \\
\hline Spin speed (r.p.m.) & Deposition time (s) & \\
\hline 3000 & 25 & 107 \\
\hline 4000 & 25 & 81 \\
\hline 5000 & 25 & 69 \\
\hline 6000 & 25 & 61 \\
\hline 7000 & 25 & 53 \\
\hline 7000 & 15 & 97 \\
\hline 7000 & 20 & 80 \\
\hline 7000 & 25 & 73 \\
\hline 7000 & 30 & 63 \\
\hline 7000 & 35 & 45 \\
\hline
\end{tabular}

Optical properties were measured using optical transmittance and reflectance spectra recorded by a UV-Vis spectrophotometer (Shimadzu UV-1800) in the 350-600 wavelength range. Electrical measurements were performed by a picoammeter/voltage source (Keithley 6487) using two planar silver (SPI ${ }^{\circledR}$ supplies) printed electrodes of $5 \mathrm{~mm}$ in length separated by $5 \mathrm{~mm}$.

\section{Results and discussion}

\subsection{Thickness measurements}

Table 1 summarizes deposition conditions of samples and their thickness. Thickness of the $\mathrm{PbI}_{2}$ thin films, in all the samples, was around of 50-100 nm. From the table, the thickness of the thin films decreased as the deposition time and spin speed increased, a typical nature of spin-coated thin films. According to spin coating theory [12], during spinning due to the centrifugal force most of the $\mathrm{PbI}_{2}$ solution is ejected from the substrate leaving a thin liquid film of uniform thickness. When the solvent, (dimethylformamide) evaporates from this film, the $\mathrm{PbI}_{2}$ deposits onto the substrate; the final thickness is achieved by the complete evaporation of the solvent [13]. Both the radial liquid flow and the solvent evaporation rate increase with increase in speed, which in turn decrease the thin film thickness, as observed in the table.

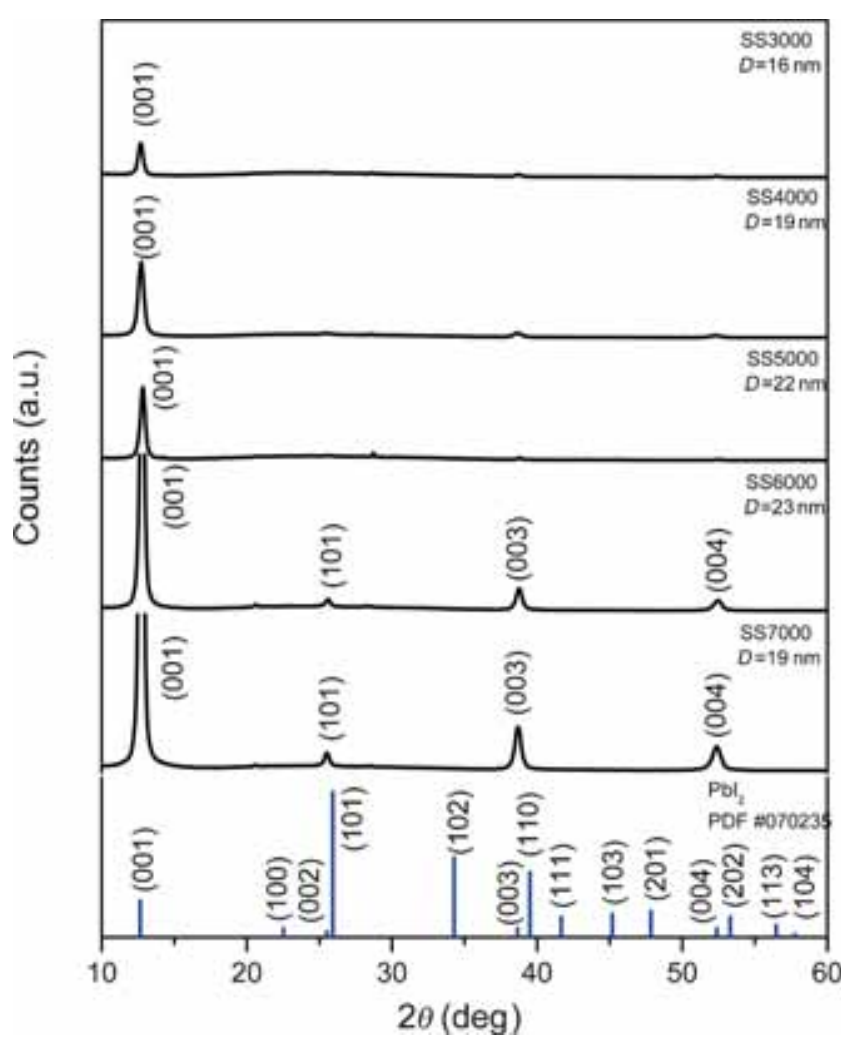

Figure 1. X-ray diffraction patterns of $\mathrm{PbI}_{2}$ thin films deposited by varying the spin speed. The standard pattern of hexagonal $\mathrm{PbI}_{2}$ (PDF 07-0235) is also included. Crystallite size calculated using the Scherrer equation for each case is noted in the respective pattern. 


\subsection{X-ray diffraction analysis}

X-ray diffraction patterns of the thin films formed by varying the spin speed are given in figure 1 . In all the patterns, the peaks present at $2 \theta=12.73^{\circ}, 38.66^{\circ}$ and $52.35^{\circ}$ were assigned to the diffraction planes (001), (101), (002) and (003), respectively, of the hexagonal crystal structure [14] (PDF 07-0235). In all the cases, crystallite size was calculated using the Scherrer equation [15]:

$$
D=\frac{0.9 \lambda}{\beta \cos \theta},
$$

where $\lambda$ is the wavelength of the X-ray beam, $\beta$ the fullwidth at half maximum and $\theta$ the Bragg's angle. The values were in the range of 16-23 nm, as shown in the figure.

In figure 2, X-ray diffraction patterns of the films deposited for various durations are given. Similar to the thin films formed at various spin speed, all the diffraction peaks were identified as reflections from (001), (110), (202) planes of hexagonal crystal structure (PDF 07-0235), as noted in the

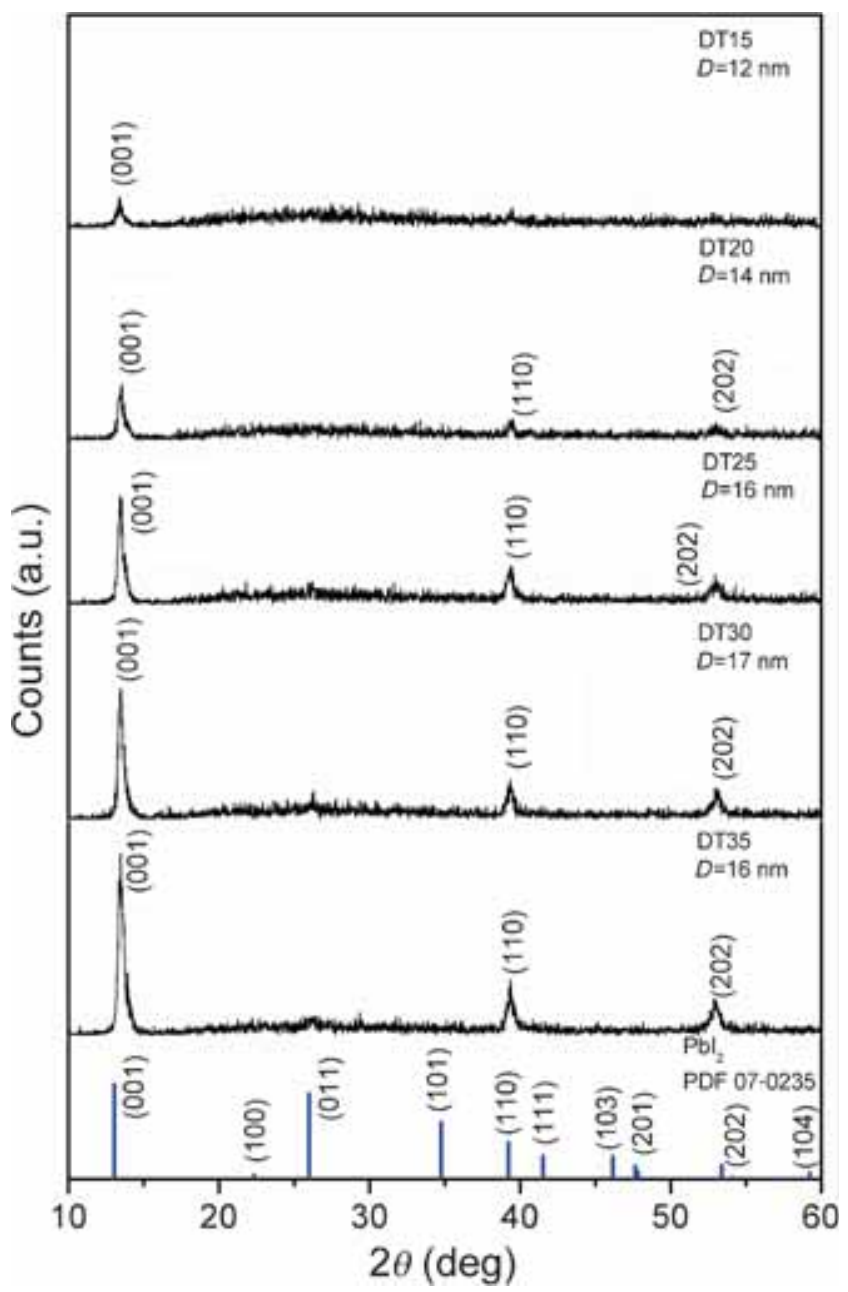

Figure 2. X-ray diffraction patterns of $\mathrm{PbI}_{2}$ thin films deposited by varying the deposition time. The standard pattern of hexagonal $\mathrm{PbI}_{2}$ (PDF 07-0235) is also included. Crystallite size calculated using the Scherrer equation for each case is noted in the respective pattern. figure. In this case, the peaks show a slight shift in the Bragg angles with respect to the standard pattern. This effect can be attributed to the presence of solvent molecules in the thin films. $\mathrm{PbI}_{2}$ is composed of stacking layers of lead and iodine atoms, traces of dimethylformamide molecules may be accommodated in between these layers. The crystallite sizes were in the range of $12-17 \mathrm{~nm}$ for the $\mathrm{PbI}_{2}$ thin films deposited for different durations at a spin speed of 7000 r.p.m., as noted in the figure. These XRD results showed that in general the thin film growth was oriented along [001] direction irrespective of the spin parameters. Formation of such highly oriented crystalline $\mathrm{PbI}_{2}$ thin films may be because of the use of dimethylformamide, a less soluble, polar solvent containing electronegative oxygen atoms. Typically, the dissolution of inorganic $\mathrm{PbI}_{2}$ in an organic solvent depends on the coordination between $\mathrm{Pb}^{2+}$ and electronegative oxygen atom in the solvent molecules showing its solubility in different extents, depending on the solvent type. The commonly used solvents are dimethylformamide, dimethylacetamide, dimethylsulphoxide and $\gamma$-butyrolactone. Among them, the slowest crystallization of $\mathrm{PbI}_{2}$ may be in dimethylsulphoxide due to its largest solubility resulting from the strongest coordination of sulphoxide oxygen with $\mathrm{Pb}^{2+}$ [16]. In a previous report on the formation of lead perovskite thin films using spincoated $\mathrm{PbI}_{2}$ precursor thin films, the role of solvents was discussed [16]. In the report, a comparative study was done on spuncoated $\mathrm{PbI}_{2}$ thin films formed using dimethylformamide and dimethylsulphoxide as solvents. The thin films formed using dimethylsulphoxide exhibited an amorphous character, whereas highly crystalline thin films were obtained

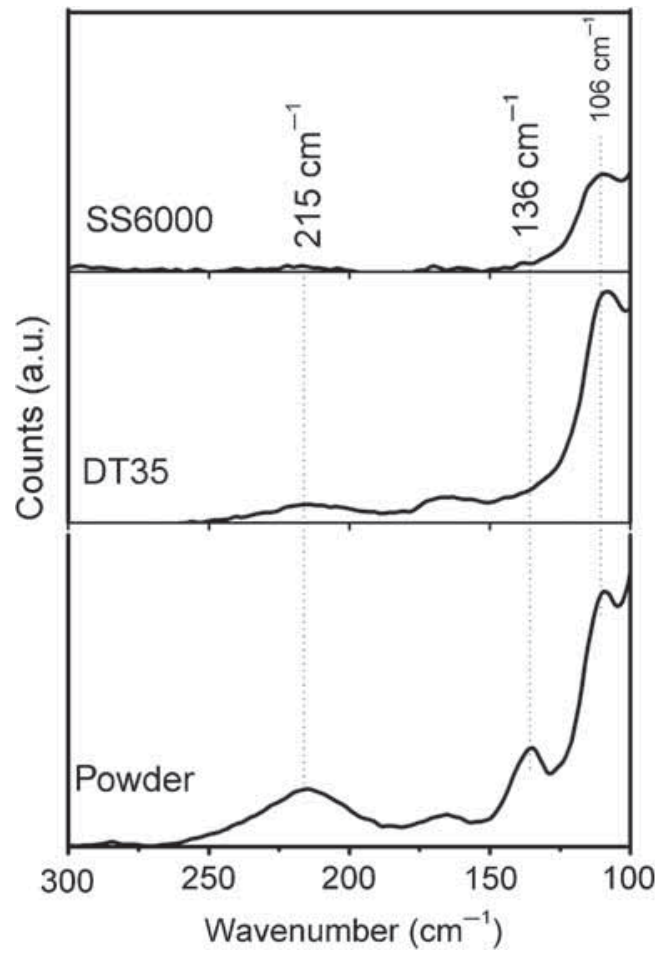

Figure 3. Raman spectra of the $\mathrm{PbI}_{2}$ thin films (samples SS6000, DT35) and the powder. 
in the case of dimethylformamide. In our experiments using dimethylformamide as solvent, hexagonal-structured $\mathrm{PbI}_{2}$ thin films oriented along [100] direction were grown

\subsection{Raman spectroscopy}

In figure 3, the Raman spectra for the typical samples SS6000, DT35 and $\mathrm{PbI}_{2}$ powder are shown. In all the spectra three peaks are present at wavenumbers 96,136 and $215 \mathrm{~cm}^{-1}$ as marked in the figure. All the peaks were identified as that originated from vibrational modes of $\mathrm{PbI}_{2}$ comparing with the reported Raman peaks for this compound [17]. As mentioned earlier, $\mathrm{PbI}_{2}$ exists in different polytypes in which the common form is $2 \mathrm{H}^{8}$ Each plane of $\mathrm{I}$ and $\mathrm{Pb}$ atoms is an identical series of triangles or overlapping hexagons. The unit cell of $2 \mathrm{H}$ contains three atoms. There are nine normal modes of vibration for the $\mathrm{PbI}_{2} 2 \mathrm{H}$ polytype, which can be represented in the irreducible $\mathrm{D}_{3 \mathrm{~d}}$ point group: $\Gamma=\mathrm{A}_{\mathrm{lg}}+$ $\mathrm{E}_{\mathrm{g}}+2 \mathrm{~A}_{2 \mathrm{u}}+2 \mathrm{E}_{\mathrm{u}}$, where $\mathrm{E}_{\mathrm{u}}$ and $\mathrm{E}_{\mathrm{g}}$ are doubly degenerate. There are three branches of acoustic modes and six branches of optical modes. The band at $96 \mathrm{~cm}^{-1}$ corresponds to the $\mathrm{A}_{\mathrm{lg}}$ mode, and that at $136 \mathrm{~cm}^{-1}$ may be due to the resonant optical excitation of the $\mathrm{PbI}_{2}$ molecules. The broadband at $215 \mathrm{~cm}^{-1}$ was also reported as a characteristic peak of $\mathrm{PbI}_{2}$ [17]. The well-defined principal band at $96 \mathrm{~cm}^{-1}$ for both the thin film samples implies their good crystalline nature in correlation with the well-defined XRD peaks. The intensity difference of the band may be due to the difference in their thickness: SS6000 (low-intensity peak-thickness $61 \mathrm{~nm}$ ) and DT35 (high-intensity peak-thickness $73 \mathrm{~nm}$ ).

\subsection{Composition}

The chemical composition of the thin films was determined using the XPS analysis. Figure 4 shows the XPS survey spectra recorded before and after etching of the thin films deposited on FTO-coated glass substrates. In this case, the thin films were deposited on conductive substrates to avoid the surface charging effect. In the survey pattern, signals corresponding to $\mathrm{C}, \mathrm{Pb}, \mathrm{I}$ and $\mathrm{O}$ are present, as marked in the figure. $\mathrm{C} 1 \mathrm{~s}$ signal was due to the adventitious carbon from the sample surface, which disappeared after Ar ion etching using argon ion of $2000 \mathrm{eV}$ energy for $5 \mathrm{~s}$. The presence of oxygen signal was from the substrates.

Figure 5 shows the high-resolution core level spectra for $\mathrm{Pb} 4 \mathrm{f}$ and I $3 \mathrm{~d}$ peaks. The peaks were de-convoluted using Shirley-type background calculation and peak fitting using Gaussian-Lorentzian sum function, as shown in the figure. The black line represents the experimental data, and the red line shows the fitted curve. Fitting was done by keeping the separation energy $(\Delta E)$ and the intensity ratio of $\mathrm{Pb} 4 \mathrm{f}$ doublet peaks at $4.87 \mathrm{eV}$ and 3:4, respectively, as shown in the figure. The peak binding energy values of $\mathrm{Pb} 4 \mathrm{f}_{7 / 2}$ and $4 \mathrm{f}_{5 / 2}$ core levels were 138.37 and $143.24 \mathrm{eV}$, in agreement with the respective values for $\mathrm{PbI}_{2}$ reported elsewhere [18]. The peak binding energy values of $\mathrm{I} 3 \mathrm{~d}_{5 / 2}$ and $3 \mathrm{~d}_{3 / 2}$ core levels

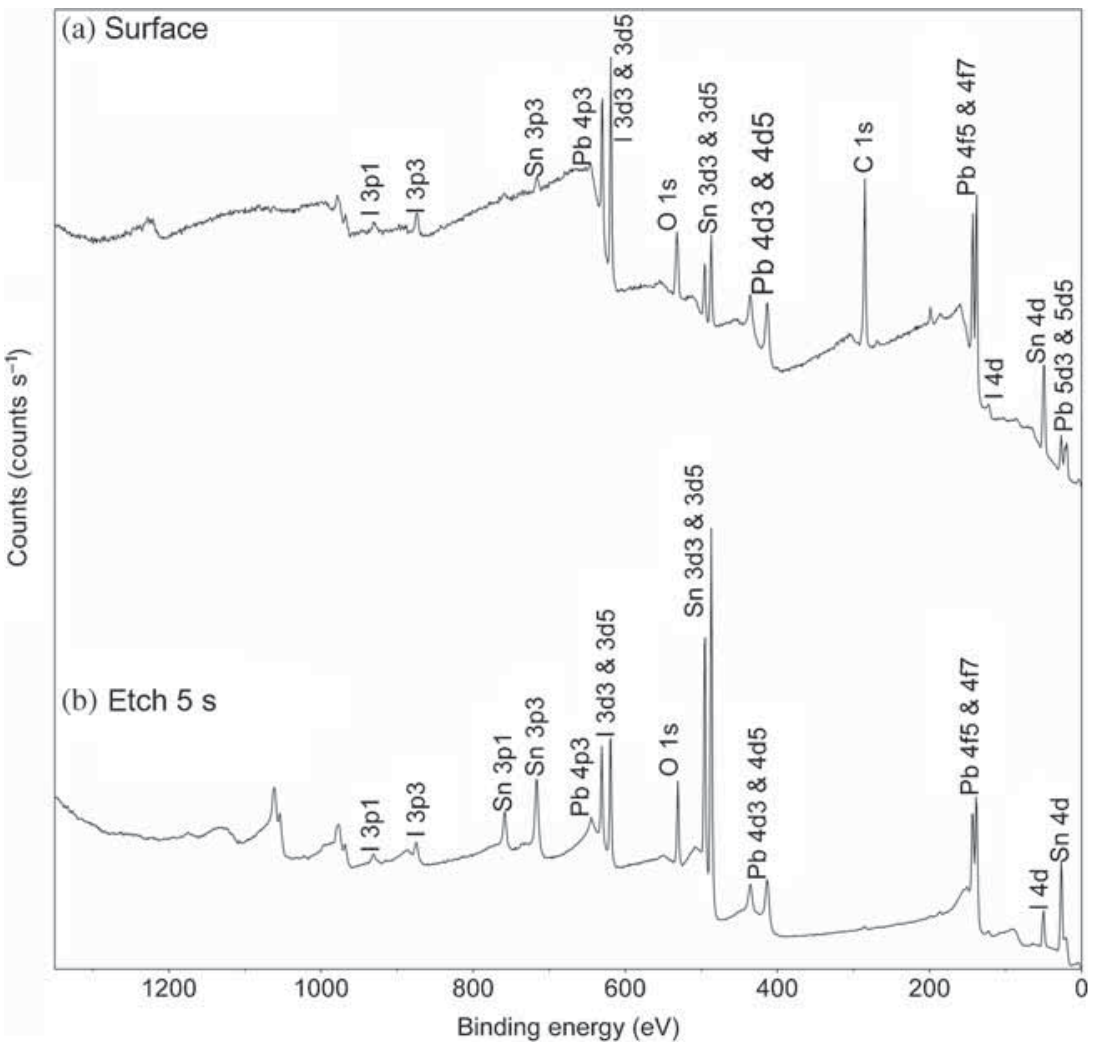

Figure 4. Survey spectra of a typical $\mathrm{PbI}_{2}$ thin film: (a) sample surface and (b) after etching. 
were 619.5 and $630.98 \mathrm{eV}$, respectively, with the doublet separation of $11.5 \mathrm{eV}$. These values are also in agreement with the values reported for $\mathrm{PbI}_{2}$ in the literature [19]. The oxidation state for lead was +4 , meanwhile the oxidation state for iodine was -2 . Further, from the figure $\mathrm{Pb} 4 \mathrm{f}$ doublet (136.5,

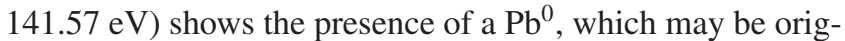
inated due to the modification of sample's surface chemistry by argon ion bombardments [20].

\subsection{Morphology}

Figure 6 shows AFM images of DT35 and SS6000 samples recorded in semi-contact mode. As shown in figure 6a, the surface morphology of DT35 consists of small grains, with an estimated roughness of $49 \mathrm{~nm}$. Sample SS6000 (figure 6b) shows a similar morphology with larger grains with an average roughness of $8.7 \mathrm{~nm}$. The difference in morphology might be attributed to the different deposition conditions for the thin films, such as spin speed and duration of deposition. For the thin films formed with higher spin speed tends to decrease the roughness, whereas increase in the deposition time leads to form more solid particle to make the morphology rougher. Such parameters may be varied to form good quality thin films.

\subsection{Optical properties}

The optical transmittance and reflectance of the thin films by varying the deposition time and spin speed (DT35 and SS6000) in the wavelength range of 350-650 $\mathrm{nm}$ are shown in figure $7 \mathrm{a}$. These thin films exhibited high transmittance in the visible spectrum, as high as $70 \%$ transmittance. The thin films were uniform and specularly reflective. Both samples showed an absorption onset around $520 \mathrm{~nm}$.

Figure $7 \mathrm{~b}$ and $\mathrm{c}$ shows the absorption coefficient of both samples and the respective Tauc plots to calculate the bandgap

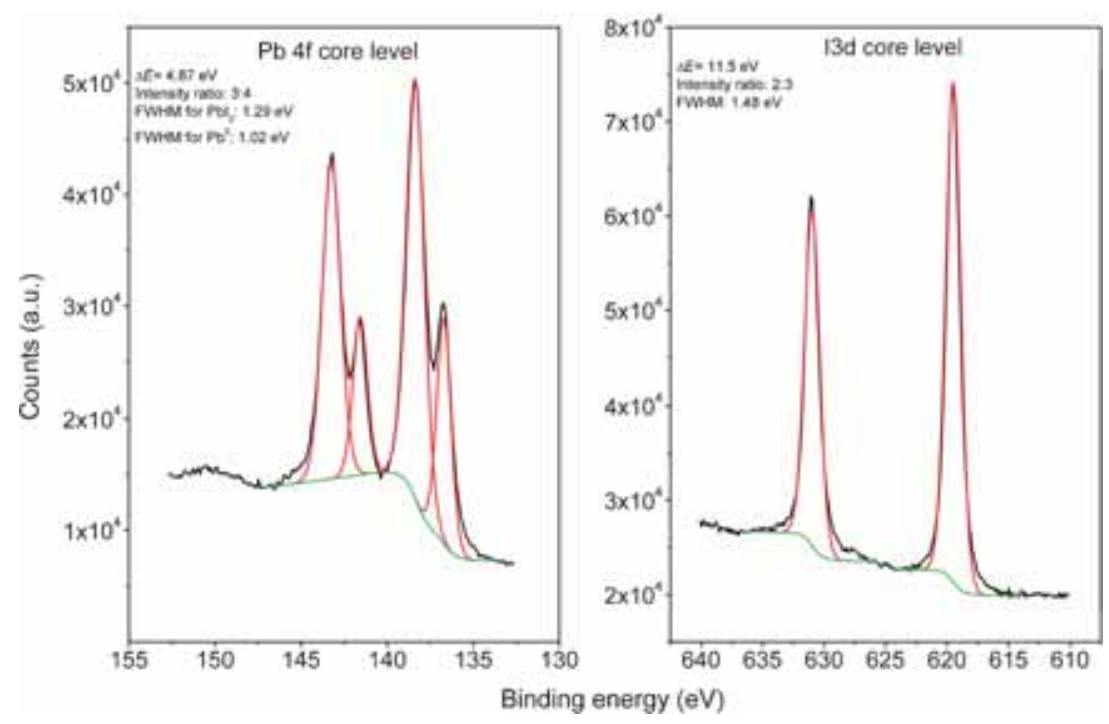

Figure 5. Core level photoelectron spectra for $\mathrm{Pb} 4 \mathrm{f}$ and I $3 \mathrm{~d}$.
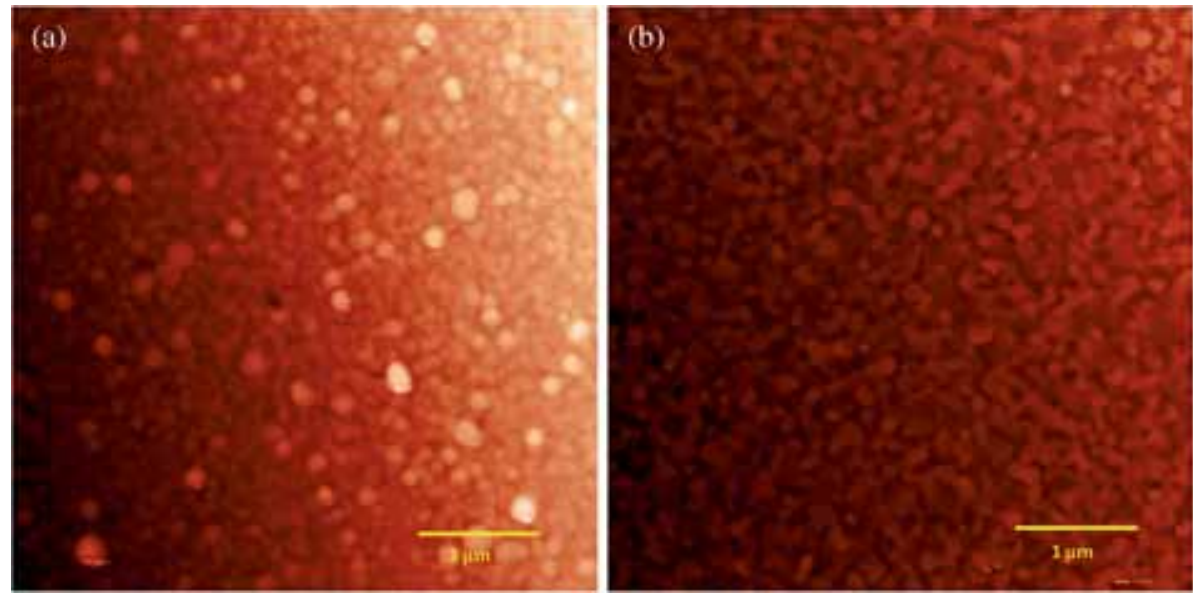

Figure 6. AFM micrographs of samples (a) DT35 (average roughness of $4.9 \mathrm{~nm}$ ) and (b) SS6000 (average roughness of $8.7 \mathrm{~nm}$ ). 
of the films. The absorption coefficient was calculated using the following equation [21]:

$$
\alpha=\frac{1}{d}\left[\operatorname{In}\left(\frac{\sqrt{(1-R)^{4}+(2 R T)^{2}}-(1-R)^{2}}{2 T}\right)\right]
$$

where $R$ is the optical reflectance, $T$ the optical transmittance and $d$ the thickness of the thin film. The samples showed very high absorption coefficients, as high as $10^{5} \mathrm{~cm}^{-1}$.

The bandgap energy of the thin films was calculated from the following equation [22,23]:

$$
(\alpha h v)^{n}=A\left(E-E_{\mathrm{g}}\right)
$$
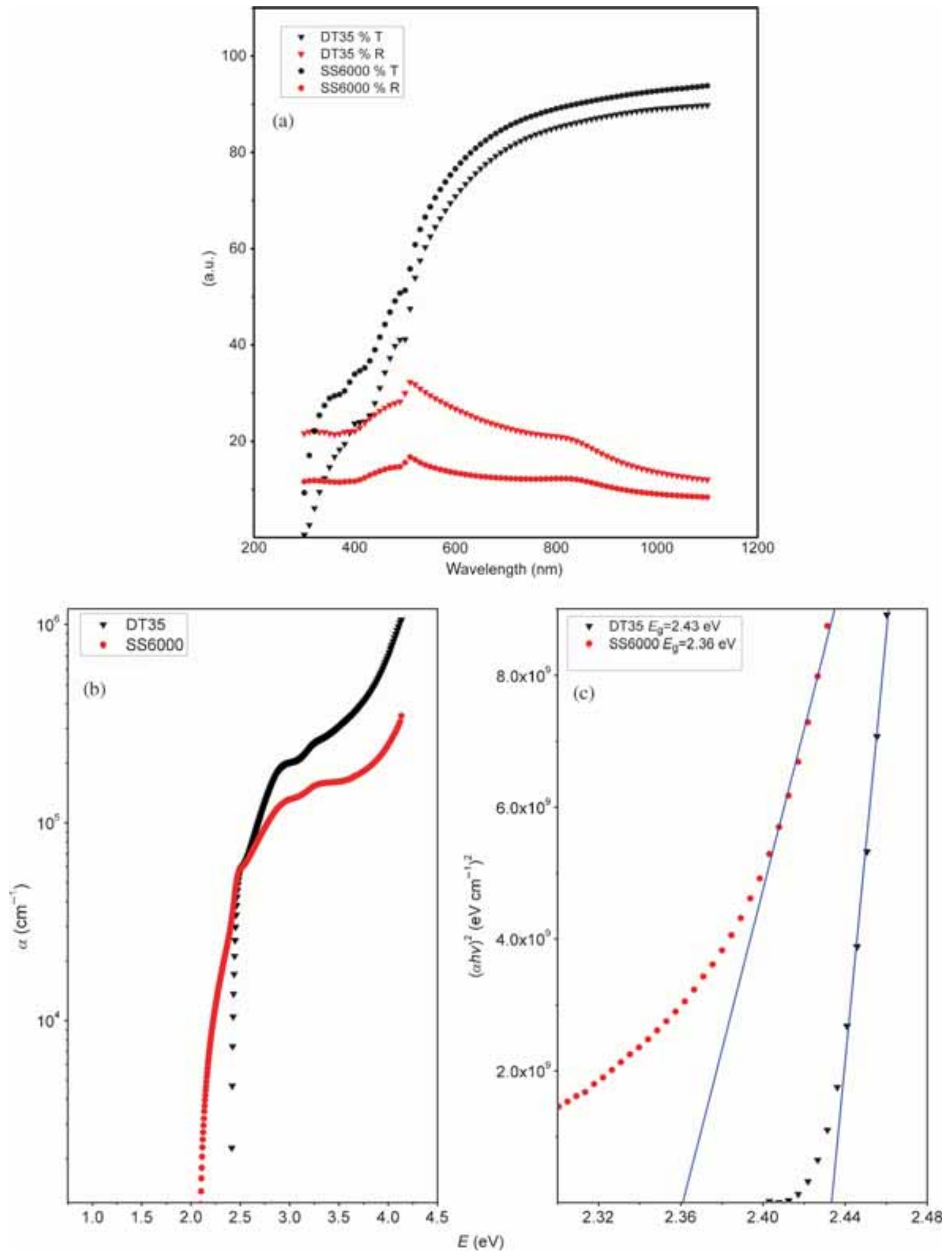

Figure 7. (a) Transmittance and reflectance spectra of the $\mathrm{PbI}_{2}$ films DT35 (7000 r.p.m. 35 s) and SS6000 (6000 r.p.m. 25 s), (b) calculated absorption spectra and (c) Tauc plots to evaluate the bandgap. 


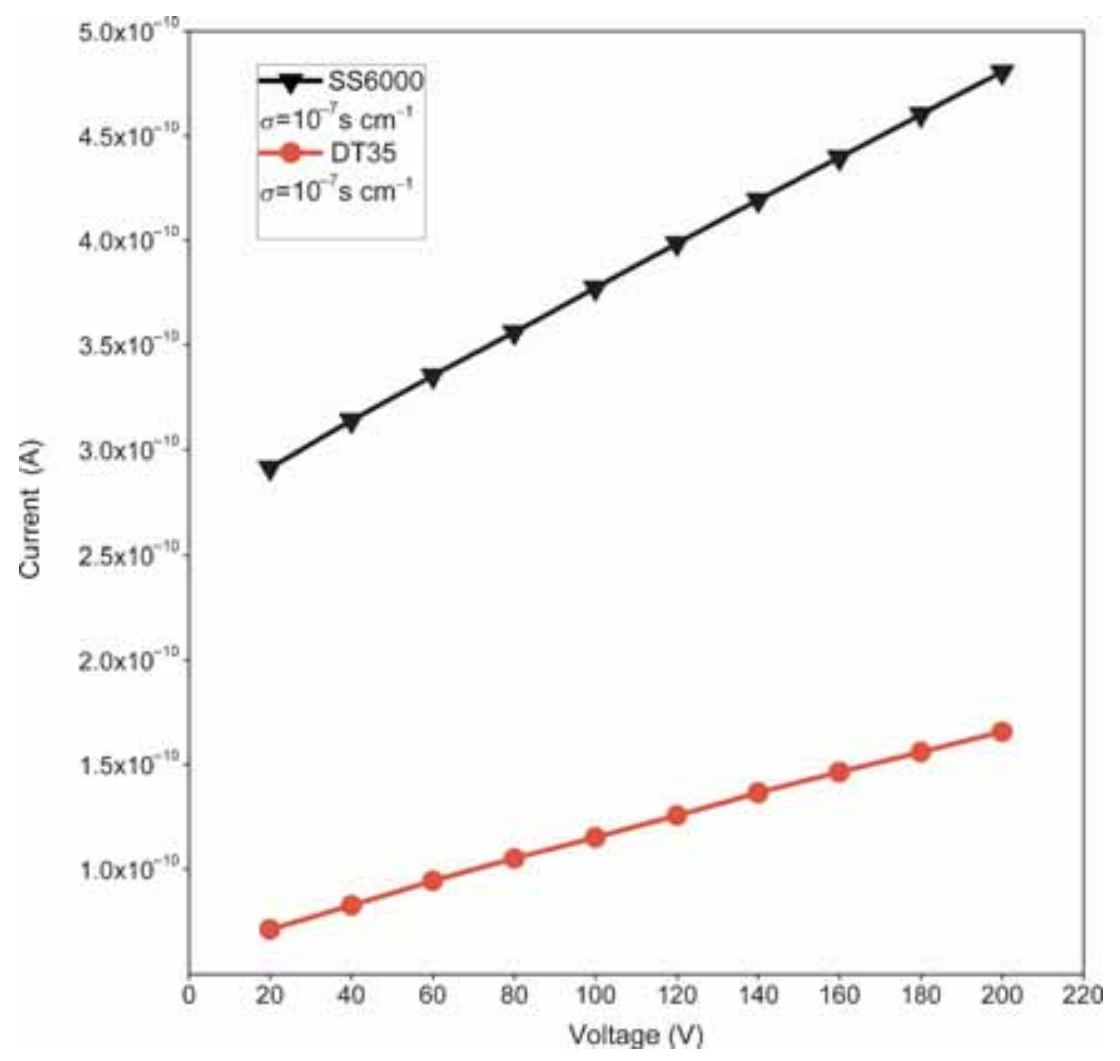

Figure 8. $I-V$ curves of the $\mathrm{PbI}_{2}$ films (samples SS6000 and DT35). The calculated conductivities are shown in the order of $10^{-7} \mathrm{~S} \mathrm{~cm}^{-1}$.

where $E_{\mathrm{g}}$ is the optical bandgap, and $n=2,1 / 2$ and $2 / 3$, respectively, for allowed direct, allowed indirect and forbidden direct transitions, $\alpha$ the absorption coefficient at frequency $v$ and constant $A$. In all the thin films, the best fit was obtained for $n=2$, corresponding to direct allowed transition. From the plot of $(\alpha h v)^{2} v s$. E, the Tauc plot, a straight line is extrapolated to E-axis giving the value of $E_{\mathrm{g}}$. The bandgap values were 2.43 and $2.36 \mathrm{eV}$ for samples DT35 and SS6000, respectively. The bandgap values decreased as thickness increased, since sample SS6000 thickness was higher than that of sample DT35. These results are in good agreement with the earlier reports [24] on $\mathrm{PbI}_{2}$ thin films deposited by different techniques.

\subsection{Electrical properties}

Figure 8 shows the $I-V$ curve for samples SS6000 and DT35. From the $I-V$ curves, a linear behaviour can be seen implying the ohmic nature of the silver contacts with the thin films. The resistivities and hence the conductivities of the thin films were calculated using the following equation:

$$
\rho=\frac{R b t}{l},
$$

where $R$ is the resistance of the sample, $b$ the width of the electrodes, $t$ the thickness of the sample and $l$ the length of the electrodes. The conductivity of the material was calculated by taking the inverse of the resistivity. Both samples exhibited conductivities of $10^{-7} \mathrm{~S} \mathrm{~cm}^{-1}$. Sample SS6000 showed a higher conductivity than that of sample DT35, which may be due to the larger crystalline size. These values of the conductivities are higher than those reported for lead iodide thin films deposited by solution methods [3].

\section{Conclusions}

Nanocrystalline thin films of lead iodide were obtained by spin coating. The thin films were uniform and polycrystalline with hexagonal structure as identified by XRD analysis, supported by the Raman spectra. The bandgap of the thin films was in the range of 2.3-2.4 eV. The thickness of the films decreased as spin speed increased. XPS measurements confirmed the elemental composition and chemical states of $\mathrm{PbI}_{2}$.

\section{Acknowledgements}

We are grateful to CeMIESOL project 35 (SENERCONACYT) Ricardo Obregon Guerra for providing the $\mathrm{X}$-ray diffraction. Daniel Acuña is especially grateful to CONACYT for providing the fellowship. 


\section{References}

[1] Lee Y and Oh K 2012 J. Instrum. 7 C0401

[2] Condeles J F and Martins T M 2004 J. Non-Cryst. Solids 33834081

[3] Ponpon J P U and Amann M 2001 Thin Solid Films 394277

[4] Novosad S S and Novosad I S 2002 Inorg. Mater. 381058

[5] Burschka J and Pellet N 2013 Nature 499316

[6] Condeles J F and Ando R A 2007 J. Mater. Sci. 43525

[7] Beckmann P A 2010 Cryst. Res. Technol. 45455

[8] Chaudhuri T K and Acharya H N 1982 Mater. Res. Bull. 17 279

[9] Mousa A M and Al-Rubaie N J 2009 Texture, Stress, and Microstructure 2009 Article ID 4945371

[10] Bhavsar D S and Saraf K B 2003 Mater. Chem. Phys. 78630

[11] Condeles J F and Mulato M 2010 J. Mater. Sci. 461462

[12] Caldeira Filho A M and Mulato M 2011 Nucl. Instr. Meth. Phys. Res. A 63682

[13] Van Hardeveld R M and Wieldraaijer W 1995 Appl. Surf. Sci. 84339
[14] Hall D B and Underhill P 1998 Polym. Eng. Sci. 38 2039

[15] Birkholz M 2006 Thin film analysis by X-ray scattering (Weinheim, Germany: Wiley-VCH Verlag GMBH and Co)

[16] Wu Y, Islam A, Yang X, Qin C, Liu J, Zhang K, Peng W and Han L 2014 Energy Environ. Sci. 72934

[17] Baibarac M, Smaranda I, Scocioreanu M, Mitran R A, Enculescu M, Galatanu M and Baltog I 2015 Mater. Res. Bull. 70762

[18] Pederson L R 1982 J. Electron Spect. Rel. Phen. 28203

[19] Zheng Z and Wang S 2007 J. Cryst. Growth 308398

[20] Moulder J F, Stickle W F, Sobol P E and Bomben K D 1992 Handbook of X-ray photoelectron spectroscopy (MN: Physical Electronics Division, Perkin Elmer Corp.)

[21] Fox M 2001 Optical properties of solids (New York: Oxford University Press Inc.)

[22] Ahmad A and Saq' an S 2009 Physica B 4041

[23] Garza J G and Shaji S 2011 Appl. Surf. Sci. 25710834

[24] Shkir M and Abbas H 2012 J. Phys. Chem. Solids 73 1309 